\title{
The Existance of the Liquid Area on the Cornea for Glaucoma Determination Using 3D-Haar Filter
}

\section{Jamal Fathi}

Department of Electrical and Electronics Engineering, Near East University, Nicosia, Northern Cyprus, Turkey

\begin{abstract}
Assessing the foremost chamber edge with gonioscopy is a vital piece of assessing patients at danger of contracting glaucoma and neovascularization. It additionally gives valuable data to patients with iris variations from the norm or a background marked by injury or visual irritation. Lamentably, gonioscopy is underutilized by both optometrists and ophthalmologists. Many manifestations call for performing gonioscopy. A standout amongst the most well-known explanations behind performing gonioscopy is in situations where the specialist suspects a patient is at danger of being tainted with Angle closure glaucoma (ACG) with understudy dilation. This paper goes for figuring the point of transparent glaucoma as a first stride of deciding the fluid's thickness accessible on the cornea, which has been ended up being extremely useful in counteracting vision misfortune. The systems included in this study incorporate proposing an eye division handle, the Angle Open Distance (AOD), Trabecular-Iris Angle (TIA), and trabecular iris space Angle (TISA). The reproduction results got and the execution of the proposed strategy are observed to be exceptionally effective and precise.
\end{abstract}

Keywords: Gonioscopy angle; Open glaucoma; Anterior chamber angle; Angle closure glaucoma (ACG); Wavelet transform; Haar filter

\section{Introduction}

The cornea is the external, straightforward, vault like structure that covers the iris, student and the foremost chamber. The iris is the hued ring of tissue suspended behind the cornea and instantly before the lens; it manages the measure of light adjusting so as to enter the eye the student's span. Open-point glaucoma, additionally called interminable glaucoma, is the thing that the dominant parts of glaucoma patients experience the ill effects of. In this condition, watery liquid does not empty well from the eye. One hypothesis is that the trabecular meshwork channel may have minuscule particles stopping up it and easing off smooth motion. Shut point glaucoma, likewise called intense glaucoma, is an ophthalmologic crisis. This happens when the iris bows forward and totally squares liquid access to the trabecular meshwork completely. The weight develops in a positive input circle, the patient has intense eye torment, and vision is lost rapidly. Intense tight point glaucoma happens all of a sudden, when the hued segment of the eye (iris) is pushed or pulled forward. These reasons blockage of the seepage edge of the eye, where the trabecular meshwork permits surge of liquids. At the point when inner eye structures are obstructed along these lines, the eyes' inside weight intraocular pressure (IOP) may spike and perhaps harm the optic nerve that transmits pictures from the eye to the cerebrum. Intense point conclusion shut edge or slender edge glaucoma produces side effects, for example, eye torment, cerebral pains, coronas around lights, widened understudies, vision misfortune, red eyes, queasiness and spewing. These signs may keep going for a considerable length of time or until the IOP is decreased. With every limited point glaucoma assault, some piece of the fringe vision may be lost. Continuous estimations of the fluid thickness on the cornea and IOP by and large can help in averting vision misfortune if treated early.

Basically, the Van Herrick system is performed keeping in mind the end goal to assess the foremost's profundity chamber. It is likewise utilized as a strategy for deciding the need of goniscopy for a specific case, which is key before widening the understudy. The Van Herrick's edge is ascertained in the biomicroscope utilizing the reference 60 degrees partition in the middle of placing so as to brighten and amplification arms the light emission at the fringe edges of the cornea and afterward contrasting the optic's span segment with the shadow between the back cornea and the iris, as shown in Figure 1 [1].

Table 1 shows the grades of the width of the optic sections and its considerations [2].

Another basic sign for gonioscopy is to concentrate on the explanation behind (IOP) increment [1]. Gonioscopy is not just used to recognize transparent edge glaucoma by choosing the accessibility of the trabecular meshwork; it likewise helps in distinguishing the explanation behind open point glaucoma. Patients with pain dysfunction syndrome (PDS) are inclined to have a regressive scratching of the iris root and a bended iris making extended contact between the back iris and the chief zonules. Contact with the zonules results in the gathering of shading



Figure 1: Van Herrick angle estimation [1].

*Corresponding author: Jamal Fathi, Department of Electrical and Electronics Engineering, Near East University, Nicosia, Northern Cyprus, Turkey, Tel: 00905338658472; E-mail: jamalfathi2004@gmail.com

Received September 26, 2015; Accepted October 17, 2015; Published October 21, 2015

Citation: Fathi $\mathrm{J}$ (2015) The Existance of the Liquid Area on the Cornea for Glaucoma Determination Using 3D-Haar Filter. J Comput Sci Syst Biol 8:6 327332. doi: $10.4172 /$ jcsb. 1000207

Copyright: @ 2015 Fathi J. This is an open-access article distributed under the terms of the Creative Commons Attribution License, which permits unrestricted use, distribution, and reproduction in any medium, provided the original author and source are credited. 
from the back iris, which is then spared all through the principal piece. Transillumination in the midperipheral locale of the iris, shade on the back surface of the cornea (Krukenberg hub), and extended shading in the trabecular meshwork are trademarks of PDS, as shown in Figure 2 [3].

Gonioscopy is a decent approach to look at anomalies of the iris and facilitates detecting iris cysts and injuries, as shown in Figure 3. Hypertension can harm the vessels (little veins) in the retina, which can hinder the flow of oxygen to the eye tissue bringing about eye sickness. An area of this monograph presents the issues distinguished amid the front's examination eye part with respect to inspecting the eye division process and the first chamber volume. These are among the key issues, which have not yet been made available in applications in present day tomographs. These calculations are either not unreasonable at all or not totally automated. The estimations in the following segments totally resolve the issue indicated and additionally show other handy ways to deal with focus it.

The rest of the paper is organized as follows. In Section II, we survey the angle width and the open angle glaucoma segmentations. In Sections III and IV, a new method of determining the thickness of the cornea is introduced and the simulation detailed design scheme is presented. Simulation results and performance evaluation are described in Section IV. Finally, Section V concludes this paper and presents future directions.

\begin{tabular}{|c|c|}
\hline Chamber Depth in Corneal Thickness & Angle Grade \\
\hline$\geq$ Cornea & 4 \\
\hline $1 / 4$ to $1 / 2$ & 3 \\
\hline $1 / 4$ & 2 \\
\hline$<1 / 4$ & 1 \\
\hline Slit & Dangerously Narrow \\
\hline
\end{tabular}

Table 1: Van Herrick angle depth estimation [2].

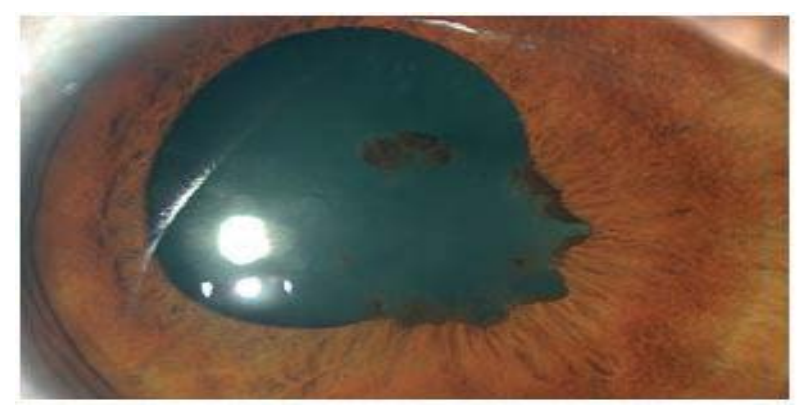

Figure 2: A photograph of the right eye showing multiple iris-lens synechiae (abnormal adhesion of the posterior surface of the iris to the anterior lens capsule).



Figure 3: Gonioscopy to appreciate elevation of iris abnormalities such as this iris cyst [1].

\section{Related Work}

Numerous specialists have introduced a few deals with the subject of recognizing/examining the veins in the eye as a first stride for glaucoma discovery. The accompanying are a present's percentage meets expectations in the eye's field veins recognition, where it happens when the vein breaks just underneath the pleasing surface of the eye. Most electronic vein location work comprises of a progression of picture handling procedures, as the retinal picture is by and large the fundamental info for examination in such research work. Creators proposed a novel strategy to concentrate vitality appropriation over wavelet sub-band marks utilizing 2D wavelet change and passed these marks to diverse element positioning and highlight choice methodologies [4]. The vitality acquired from point by point coefficient is utilized to group ordinary and glaucomatous picture with high exactness. This was grouped utilizing bolster vector machines, successive insignificant streamlining, arbitrary timberland, innocent bayes and fake neural system (ANN). The work accomplished an exactness of $94 \%$ utilizing the ANN classifier. Nandibewoor et al. proposed component extraction of individual's eye shading varieties taken by top quality laser camera and called them fundus pictures, did utilizing MATLAB programming apparatus [5]. By measuring the shading pixels in the influenced range the perception demonstrated that the individual is experiencing glaucoma or not. Likewise, a test was made utilizing the picture of an ordinary individual which was kept as reference and after that contrasted and the clinical perceptions of the individual's picture. Khalid et al. proposed the sending of widening and disintegration with Fuzzy c-Means (FCM) as a powerful optic glass and circle division [6]. The least expensive approach to screen glaucoma illness is utilizing advanced fundus camera. These pictures were put away in RGB group which were part into red, green and blue channels. The extricated green channel was portioned with FCM. The division was assessed in view of the ground truth regions that were laid out by the ophthalmologists. The glass to-circle proportion (CDR) estimations were ascertained from the distance across proportion of the fragmented container and plate. Hussain et al. represented a framework which was mostly in light of picture handling and comparing so as to group strategies for programmed discovery of glaucoma and measuring distinctive parameters of fundus pictures of glaucoma patients and typical patients [7]. Padaria et al. gave a review of distinctive systems for optic container and optic circle identification from retinal picture [8]. The Optic Cup (OC) is subject to the sort of glaucoma and the level of intraocular weight to rearrange the division process. Abirami et al. proposed an information center based fluffy min-max neural system (DCFMN) to identify and order glaucoma, typical pictures, and the stratus Anterior Segment Optical Coherence Tomography (ASOCT) pictures. DCFMN contains two classes of neurons: arranging neurons (CNs) and covering neurons (OLNs) [9]. CNs was utilized to characterize the examples of information, and the OLNs could deal with a wide range of cover in diverse hyper boxes. In this work, another sort of participation capacity considering the attributes of information and the impact of clamor was intended for CNs in the DCFMN. The participation capacity of OLNs managed the relative position of information in the hyper boxes. Kavitha et al. proposed a methodology for the programmed limitation and precise limit identification of optic circle utilizing the part examination system and region of interest (ROI) based division [10]. Associated part investigation technique was utilized to recognize the optic container. The strategy was contrasted and manual thresholding methodology and later the dynamic form was utilized to plot the limit precisely. This system could be utilized to naturally portion the neuro-retinal edge territory utilizing a cover 
to channel ISNT second rate, prevalent, nasal, transient quadrants. Neuro-retinal edge territory was figured in each of the quadrants independently to suspect glaucoma. Durga Devi et al. principally focused on eye pathology and indicated whether eye sickness will bring about the iris acknowledgment procedure to fall flat [11]. Iris pictures were taken prior and then afterward the treatment of eye infection and the yield demonstrated the scientific distinction acquired from treatment. Gabor channel were utilized to remove the elements. At long last, the creators inferred that the proposed iris acknowledgment ought to be utilized to tackle the potential issues that could emerge in key biometric innovation and therapeutic determination. Kim et al. assessed the adjustments in anterior chamber depth (ACD) and edge width incited by phacoemulsification and intraocular lens (IOL) implantation in typical eyes utilizing foremost anterior segment optical coherence tomography (AS-OCT) [12].

\section{Proposed Methods}

Generally, the thickness of the cornea is measured using ultrasound to get the related measurements for the values of (AOD), (TIA), and (TISA) [13]. See Figure 4 . The proposed work presents a highly efficient image processing based technique for these measurements and faster than the normal methods used nowadays as in the sonar method.

Verifying the AOD, TIA, and TISA methods sensitivity to parameters change was carried out, likewise in the previous segment, with and without semi-mechanization actualized in business programming. Self-loader stamping of point's normal for individual techniques is identified with dragging the point checked to the edge, regularly utilizing the dynamic form strategy. On the other hand, in AOD, TIA, and TISA systems the outcome is influenced by the spot demonstrated by the administrator. Preparatory estimations have affirmed, contingent upon the administrator, a lapse of point's sign of around \pm 10 pixels, giving a slip at the determination of 32 pixels/ $\mathrm{mm}$ of around $\pm 0.31 \mathrm{~mm}$. Narrowing from focuses $\mathrm{p} 1$ and $\mathrm{p} 2$ to $\mathrm{p} 3$ and $\mathrm{p} 4$ as appeared in Figure 4, takes into account the examination of affectability to the parameters. Measuring changes in AOD, TID and TISA systems as for this narrowing method is the first stride of this work. On this commence the going with suspicions related to the studies completed in ref. [14] have been shaped:

- The range of characteristic points position variability \pm 10 pixels,

- Verified software in semi-automatic version,

- Analysis, due to comparative reasons, narrowed to points $\mathrm{p}_{1}$ and $\mathrm{p}_{2}$,

- The analyzed image resolution of 32 pixels $/ \mathrm{mm}$.

- The left eye is selected for this study.

As noticed from the above Figure 4, the white spot belongs to the light reflected on the eye and it's neglected in the calculations.

The measurement error calculated for the AOD method $-\delta_{\mathrm{AOD}}$, for TIA $-\delta_{\text {TIA }}$ for TISA $-\delta_{\text {TISA }}$ will be calculated as the difference between the measured and the correct value, expressed as the percentage of notional value, where the notional value is most often understood as the correct value as shown in Equation 1.

$$
\ddot{a}_{A O D}=\frac{d_{M}-d_{W}}{d_{W}} \times 100 \%
$$

$$
\begin{gathered}
\ddot{a}_{T I A}=\frac{\dot{a}_{M}-\dot{a}_{W}}{\dot{a}_{W}} \times 100 \% \\
\ddot{a}_{T I S A}=\frac{s_{M}-s_{W}}{s_{W}} \times 100 \%
\end{gathered}
$$

Where, $\mathrm{s}_{\mathrm{M}}, \mathrm{s}_{\mathrm{W}}$ are the measured standard areas, $\alpha_{\mathrm{M}}, \alpha_{\mathrm{W}}$ are the measured standard angles, and $\mathrm{d}_{\mathrm{M}}, \mathrm{d}_{\mathrm{W}}$ are the measured standard distances.

The distance equation as shown in Equation 2 is taken into consideration.

$$
\begin{aligned}
& d_{M}=\sqrt{\left(y_{1}-y_{2}\right)^{2}+\left(x_{1}-x_{2}\right)^{2}} \\
& d_{W}=\sqrt{\left(y_{W 1}-y_{W 2}\right)^{2}+\left(x_{W 1}-x_{W 2}\right)^{2}}
\end{aligned}
$$

The sensitivity with respect to the change in parameters will be the measured value as shown in Equation 3.

$$
\delta_{A O D(p i)}=\frac{\delta d_{M}}{d_{M} \delta x_{i}} \times 100 \%
$$

Where, $x_{i}$ and $y_{i}$ are the coordinates of points $\mathrm{p}_{1}$ and $\mathrm{p}_{2}$ that were analyzed in this research. In this work, four points are taken into consideration.

$$
\begin{gathered}
\delta_{T I A(p i)}=\frac{\Delta \alpha_{M}}{\dot{a}_{M} \Delta \dot{a}_{i}} \times 100 \% \\
\delta_{T I S A(p i)}=\frac{\Delta s_{M}}{s_{M} \Delta s_{i}} \times 100 \%
\end{gathered}
$$

In the above equations, the calculations taken into consideration are the negatives of the original image to calculate the contour. A selected eye in a sample original image is shown in Figure 5 [15].

\section{Simulation}

The image database (DB) was taken from patients in a Jordanian Governmental hospital (Al Ameera Basma Hospital). The patients had ages ranging between 40 and 65 years old, and all of them had eyerelated infections or sicknesses. The proposed system is implemented as a real-time face detection and tracking system using 720P (1280 $\times 720$ )@ 30 frames per second (FPS). In this study, a sample input

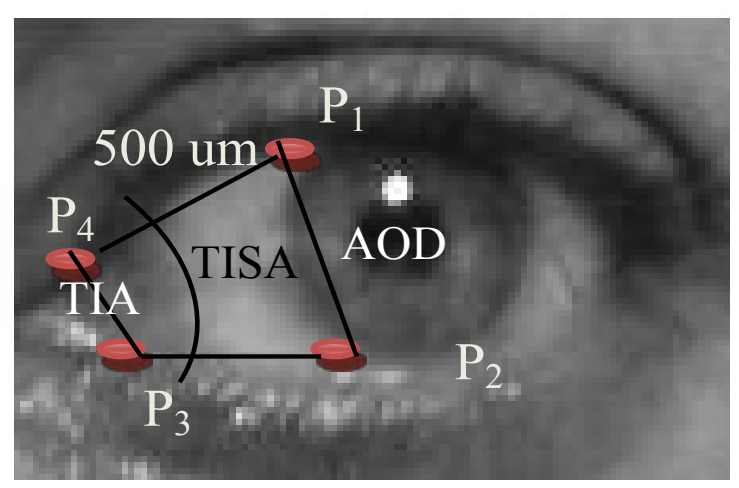

Figure 4: Location of points pi indicated by the operator. 
Citation: Fathi J (2015) The Existance of the Liquid Area on the Cornea for Glaucoma Determination Using 3D-Haar Filter. J Comput Sci Syst Biol 8:6 327-332. doi:10.4172/jcsb.1000207

picture is shown in Figure 6, which is taken from the saved database.

When every image is saved as a JPEG index, then the following procedure is applied on each image:

\section{Face selection.}

\section{Eye selection.}

3. Eye segmentation in order to obtain the contour.

The real-time face detection and tracking system is implemented using 720P $(1280 \times 720) @ 30$ frames per second $(\mathrm{fps})$ based on the Viola-Jones face detection algorithm. The color format representing the image in each frame is RGB. In this system, the Circular Hough Transform (CHT) has been adopted for iris detection. The Hough transform has been modified into several versions. It has been considered an effective method for detecting curves in images. CHT was presented by A. Herout as a modified version of the original Hough transform [16]. CHT's purpose is to recognize circular patterns in an image. It is used to transform sets of feature points residing in the image space into sets of votes that are accumulated in the parameter space.

After the above procedure is done, the selected eye is saved in a separate folder. The 3D-Haar filter is applied to cancel the eyelashes, and the noise inside the eye, and then to get the liquid material in the eye alone. The analysis filter banks decompose a port signal into frequency sub-bands. A two channel analysis filter bank, as shown in Figure 7, splits the input $\mathrm{X}(\mathrm{z})$ right into a high frequency $(\mathrm{HF})$ component $\mathrm{U}_{0}(\mathrm{z})$ along with a low frequency (LF) component $\mathrm{U} 1(\mathrm{z})$. The input $\mathrm{X}(\mathrm{z})$ is passed via a low pass filter $\mathrm{H}_{0}(\mathrm{z})$ and a high pass filter $\mathrm{H}_{1}(\mathrm{z})$, yielding the $\mathrm{U}_{0}(\mathrm{z})$ and $\mathrm{U}_{1}(\mathrm{z})$ respectively [17].

Moreover, this procedure is applied to obtain the liquid parts on the eye as shown in Figure 8, and then to get the contour image as shown in Figure 9.

Measurements were carried out changing the position of points $\mathrm{p}_{1}$ and $\mathrm{p}_{2}$ in coordinate $\mathrm{x}$ within $\mathrm{x}_{\mathrm{w}} \pm 10$ pixels, assuming automated dragging to the contour line on the $y$ axis. The obtained result is shown in Figure 10.

The results obtained admittedly show an advantage of AOD method, in which a change of point's position by the operators affects the total error to the least extent and at the same time this method is least sensitive to operators' errors, but only in cases of ideal determination of the contour. Unfortunately it turns out that in the case of disturbances,

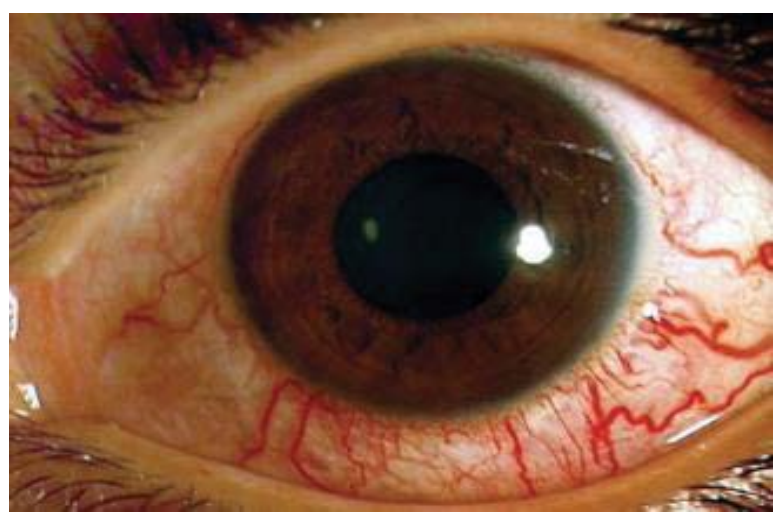

Figure 5: Selected eye of the original image.

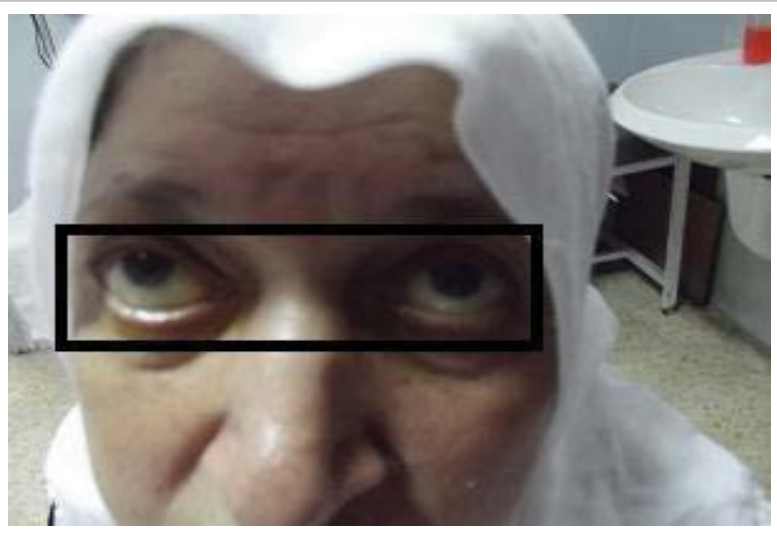

Figure 6: Original Image.

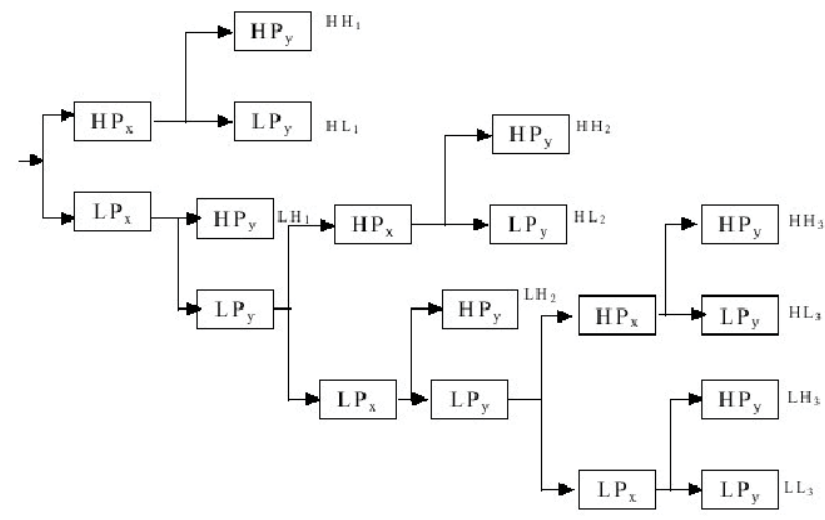

Figure 7: Three-channel Analysis filter bank [17].

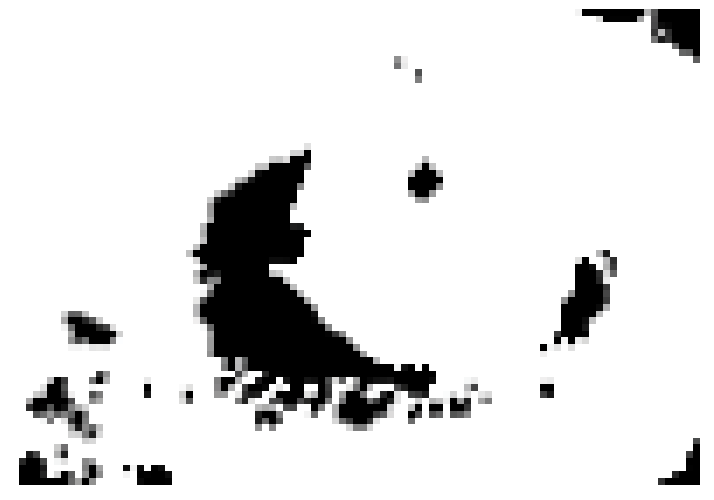

Figure 8: Negative of the original image to calculate the contour.

personal variability and other factors causing sudden local contour changes/fluctuations, the situation is slightly different, and a white noise is assumed to be added to the image with the aim of obtaining the contour. The uniform distribution is set to be \pm 20 to reduce the white noise. The obtained results are shown in Figure 11.

Taking also into consideration the medical premise, a profiled algorithm for analyzing and processing of anterior eye segment has been proposed in ref. [18]. Using the proposed calculations, the results obtained, for three methods AOD, TIA and TISA, for error value and 




Figure 9: Obtained contour.

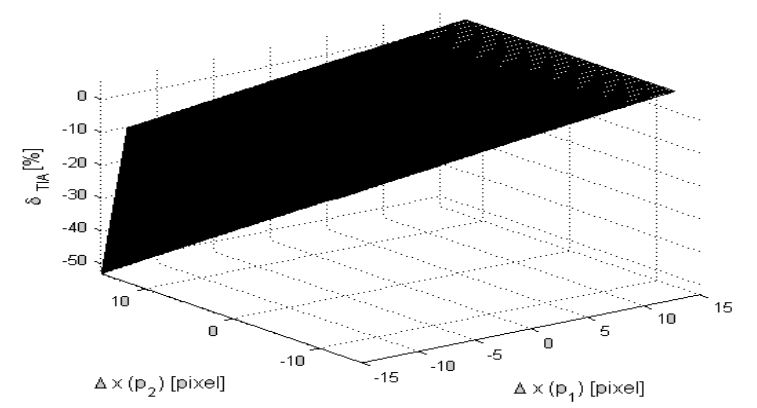

Figure 10: Obtained changes in the error values $\delta$ TIA versus the position of the points on the axis.

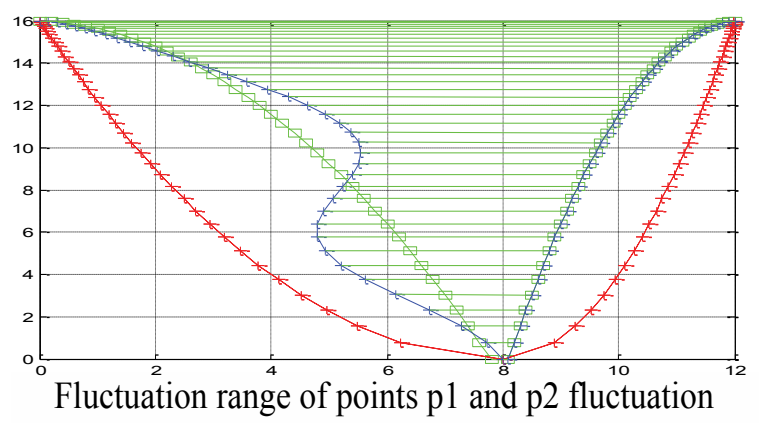

Figure 11: This Figure shows the contour, where the uniform distribution is set to be \pm 20 .

sensitivity to changes in points $\mathrm{p}_{1}$ and $\mathrm{p}_{2}$ position are tabulated in Table 2 .

The findings showed that the obtained results in comparison with angle width in open and closed glaucoma calculated in ref. [19] were similar. This yields that the proposed method is faster, easy to use, and accurate in performance. The obtained results in ref. [19] are tabulated in Table 3 as a reference.

The three methods AOD, TISA and TIA are applied on the database taken from the patients and the obtained results are shown in Figure 12.

The results obtained during the process of filtration clearly demonstrate an advantage of the AOD method, in which a change of points' position by operators affects the total error to the least extent. This method is at the same time less sensitive to operator errors, but only in cases of ideal determination of the contour. Unfortunately, in the cases of disturbances, personal variability and other factors causing sudden local contour changes/fluctuations, the situation is slightly different. Plots of AOD, TIA, and TISA are shown in Figure 13.

As can be seen in Figures 12 and 13 and has already been stated, the AOD strategy does not adapt well to cases where the patient has eye abnormalities. The new proposed strategy works by scanning the area of the eye shown in Figure 4, to calculate AOD, TIA, and TISA values at 1 pixel intervals (where the AOD line is pushed closer to the TIA by 1 pixel), the intervals were stopped once the borders of the liquid were reached. The means of the AOD, TIA, and TISA were then calculated. One case was evaluated by the proposed method, and it was found that it took on average 16 intervals to reach the borders of the liquid, the typical resolution of the image was found to be 32 pixels $/ 1 \mathrm{~mm}$. Moreover, some pixels were influenced by external and internal noise

\begin{tabular}{|c|c|c|}
\hline Method & $\mathbf{S}_{(\mathbf{p} 1)}$ & $\mathbf{S}_{(\mathbf{p 2})}$ \\
\hline AOD & $0.1007 \%$ & $-0.0002 \%$ \\
\hline TIA & $0.2783 \%$ & $0.0368 \%$ \\
\hline TISA & $0.4983 \%$ & $0.6184 \%$ \\
\hline
\end{tabular}

Table 2: Obtained results according to the changes in position.

\begin{tabular}{|c|c|}
\hline LAOD & 0.002 \\
\hline DTAOD & 0.001 \\
\hline DNAOD & 0.000 \\
\hline DAOD & 0.004 \\
\hline
\end{tabular}

Table 3: Angle width measurement [19]

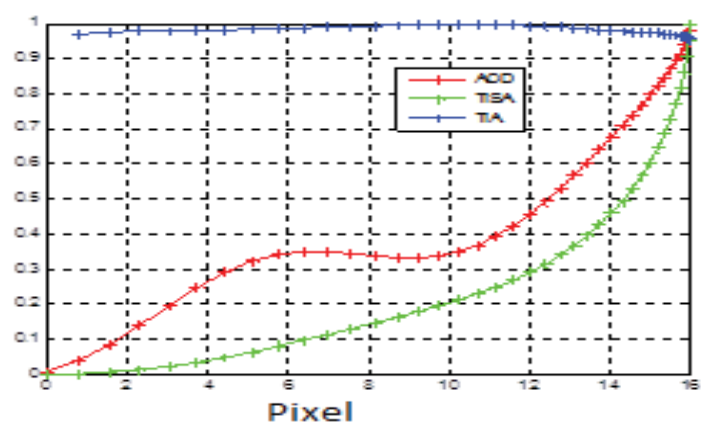

Figure 12: The Filtration angle for the three methods.
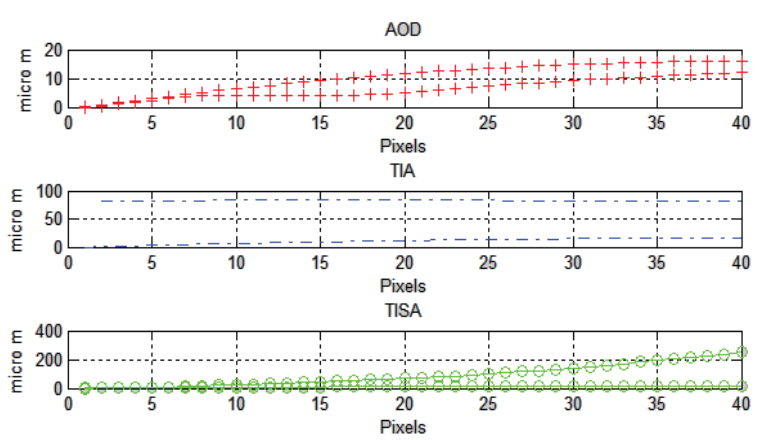

Figure 13: AOD, TIA, and TISA in micro $m$ vs. pixels. 
Citation: Fathi J (2015) The Existance of the Liquid Area on the Cornea for Glaucoma Determination Using 3D-Haar Filter. J Comput Sci Syst Biol 8:6 327-332. doi:10.4172/jcsb.1000207

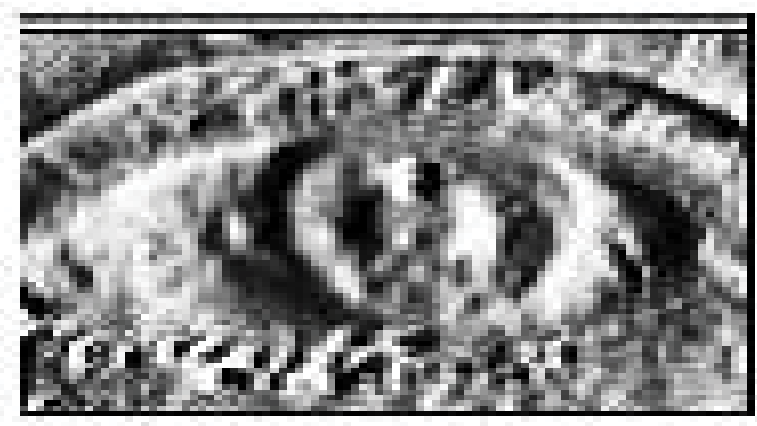

Figure 14: Obtained image after the filtration process.

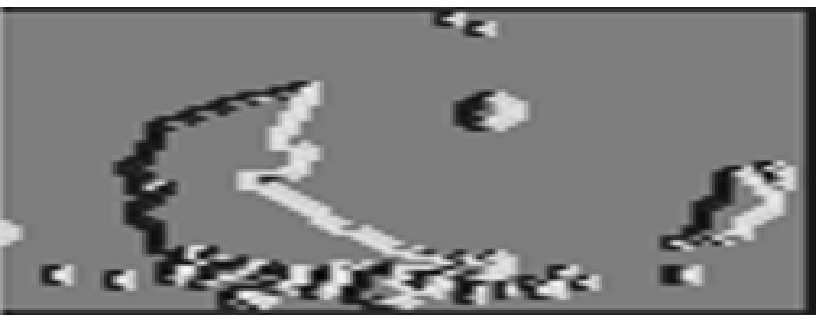

Figure 15: The liquid area available on Cornea after applying Haar Filter.

such as the lighting of the room, and the eyelashes respectively. This method of estimation is affected by variables mentioned previously, but once those variables have been corrected for using the Haar filter, the technique is exact and effective. The images after applying the 2D Haar filter are shown in Figures 14 and 15.

\section{Conclusion}

Thickness of the liquid available on the Cornea has revolutionized the assessment of the foremost portion of the eye. The structures encompassing the posterior chambers which were hard to analyze clinically, are now being imaged and evaluated in subtle element. The utilization of this innovation gives a magnificent perspective of the pathology happening in the front and back councils of the eye and permits target documentation of the foremost chamber point and the ciliary body, giving a reasonable knowledge into the reason for fluid block. It additionally helps in identifying a glaucoma case as well as determining the need for surgery.

\section{References}

1. Dabasia PL, Edgar DF, Lawrenson JG (2013) Measurement of the Anterior Chamber Angle, Part 2: Screening for Angle Closure and Angle Closure Glaucoma. Optometry in Practice 14: 147-154.

2. Friedman DS, He M (2008) Anterior chamber angle assessment techniques. Surv Ophthalmol 53: 250-273.

3. Singh P, Tyagi M, Kumar Y, Kuldeep K, Sharma PD (2013) Gonioscopy: A Review. Open Journal of Ophthalmology 3: 118- 121.

4. Rajandran N (2014) Glaucoma Detection Using Dwt Based Energy Features and Ann Classifier. IOSR Journal of Computer Engineering 16: 35-42.
5. Nandibewoor A, Kulkarni SB, Byahatti S, Hegadi R (2013) Computer Based Diagnosis of Glaucoma using Digital Fundus Images. Proceedings of the World Congress on Engineering. London, UK 3: 1-3.

6. Khalid NEA, Noor NM, Ariff NM (2014) Fuzzy c-Means (FCM) for Optic Cup and Disc Segmentation with Morphological Operation. International Conference on Robot PRIDE - Medical and Rehabilitation Robotics and Instrumentation, Conf PRIDE, Malaysia.

7. Hussain SA, Holambe AN (2015) Automated Detection and Classification of Glaucoma from Eye Fundus Images: A Survey. International Journal of Computer Science and Information Technologies 6: 1217-1224.

8. Padaria AR, Limbasiya B (2015) A Review Paper on Detection of Optic Disc Damage using Retinal Images. International Journal of Computer Applications 111: $1-4$.

9. Abirami SS, Shoba SJG (2013) Glaucoma Images Classification Using Fuzzy Min-Max Neural Network Based On Data-Core. International Journal of Science and Modern Engineering 1: 9-15.

10. Kavitha S, Karthikeyan S, Duraiswamy K (2010) Neuroretinal Rim Quantification in Fundus Images to Detect Glaucoma. IJCSNS International Journal of Computer Science and Network Security 10: 134-140.

11. Durga Devi G, Preethi DMD (2014) Disease Identification in Iris Using Gabor Filter. International Journal of Engineering and Computer Science 3: 53965399.

12. Kim M, Park KH, Kim TW, Kim DM (2011) Changes in Anterior Chamber Configuration after Cataract Surgery as Measured by Anterior Segment Optical Coherence Tomography. The Korean Ophthalmological Society 25: 77-83.

13. Fea AM, Bertaina L, Consolandi G, Damato D, Lorenzi U, et al. (2012) Angle Closure Glaucoma: Pathogenesis and Evaluation. A Review. J Clinic Experiment Ophthalmol S4: 005.

14. Dabasia PL, Edgar DF, Lawrenson JG (2014) Methods of measurement of the anterior chamber angle Part 3: Screening for angle closure and angle closure glaucoma using advanced technologies. Optometry in Practice 15: 11-18.

15. Kumar PJS, Banerjee S (2014) A Survey on Image Processing Techniques for Glaucoma Detection. International Journal of Advanced Research in Computer Engineering \& Technology 3: 4066-4073.

16. Herout A (2013) Review of Hough Transform for Line Detection A. In Real-Time Detection of Lines and Grids, Springer-Verlag London, UK.

17. Talukder KH, Harada K (2007) Haar Wavelet Based Approach for Image Compression and Quality Assessment of Compressed Image. IAENG International Journal of Applied Mathematics 36: 1-8.

18. Tian J, Marziliano P, Baskaran M, Wong HT, Aung T (2011) Automatic anterior chamber angle assessment for HD-OCT images. IEEE Trans Biomed Eng 58: 3242-3249.

19. Masoodi H, Jafarzadehpur E, Esmaeili A, Abolbashari F, Ahmadi Hosseini SM (2014) Evaluation of anterior chamber angle under dark and light conditions in angle closure glaucoma: An anterior segment OCT study. Cont Lens Anterior Eye 37: 300-304. 